\section{Life expectancy in patients with inflammatory bowel disease: time will tell if biologics are the answer}

We thank Kuenzig and colleagues for showing that, despite an upward trend in life expectancy in patients with inflammatory bowel disease (IBD), both men and women with IBD still die younger and have worse overall pain and emotional well-being scores than matched controls without IBD. ${ }^{1}$ These results align with a recent Swedish cohort study that showed that children with IBD were 3 times more likely to die than those without IBD. ${ }^{2}$ Given that the prevalence of IBD is increasing worldwide and, in Canada, is expected to reach $1 \%$ within the next 5 years, ${ }^{3}$ how can we now narrow this gap in life expectancy?

Nowadays, at least one-third of patients with IBD, most often those with severe and widespread disease, are treated with biologic medication. In Canada, although anti-tumour necrosis factor therapy has been licensed for almost 2 decades, biologics were not widely prescribed until around $2010 .{ }^{4}$ Kuenzig and colleagues assessed medication use in patients older than 65 years of age to 2011 only; treatment strategy, in the context of disease phenotype and severity, was not available in the authors' administrative data set. Stratification of data relative to these factors would have helped to better understand the impact of biologics on the lived experiences of patients, which, as the authors point out, was worse than in non-IBD matched controls.

The use of biologics has increased substantially since the end date of Kuenzig and colleagues' study, facilitated in part by the widespread adoption of biosimilars. Therefore, their results are already a little outdated. Given recently published data regarding patients with IBD on biologics that support decreased rates of hospital admission and surgery, with improvement in quality of life, the analysis should be repeated soon. ${ }^{5,6}$ The established positive impact of biologics on disease activity should be weighed against the associated profile of adverse effects, as well as any emerging findings regarding life expectancy. ${ }^{7}$

Kuenzig and colleagues report improvement over time in the overall life expectancy of patients with IBD, but this was not paralleled by improved health-adjusted life expectancy (HALE). Although changes in the proportion of patients on biologics may also help reduce the amount of inflammatorymediated symptoms, these changes may not necessarily improve HALE. The assessment and management of pain, fatigue and emotional well-being in patients with IBD is complex and poorly understood, but only by dealing with these clinical problems directly is HALE likely to change. Resources to support help with this are emerging: a Cochrane systematic review comprehensively evaluating interventions for managing abdominal pain in Crohn disease is underway (CD013531) and a practical treatment algorithm on assessing fatigue in IBD has recently been published. ${ }^{8}$ Toolkits for family doctors related to diagnosing IBD, treating flares, managing complications and navigating special circumstances, such as pregnancy and cancer screening, are also available and are garnering postimplementation, real-world evidence. ${ }^{9}$

The true biologic era is now, and analysis of data regarding medication, disease activity, health care use and quality of life, collected over the last decade, will tell if the use of biologics will not only improve life expectancy but also the lived experiences of patients with IBD.

Cite as: CMAJ 2021 March 15;193:E380. doi: $10.1503 / \mathrm{cmaj} .78144$

\section{Neil Chanchlani MBChB MSc}

Gastroenterology research fellow, Royal Devon and Exeter Hospital, Exeter, UK

\section{Richard K. Russell MBChB PhD}

Professor of paediatric gastroenterology, The Hospital for Sick Children, Edinburgh, UK

\section{References}

1. Kuenzig ME, Manuel DG, Donelle J, et al. Life expectancy and health-adjusted life expectancy in people with inflammatory bowel disease. CMAJ 2020;192:E1394-402.

2. Olén O, Askling J, Sachs MC, et al. Increased mortality of patients with childhood-onset inflammatory bowel diseases, compared with the general population. Gastroenterology 2019;156:614-22.

3. Ng SC, Shi HY, Hamidi N, et al. Worldwide incidence and prevalence of inflammatory bowel disease in the 21st century: a systematic review of population-based studies. Lancet 2018;390: 2769-78.

4. Targownik LE, Tennakoon A, Leung S, et al. Temporal trends in initiation of therapy with tumor necrosis factor antagonists for patients with inflammatory bowel disease: a population-based analysis. Clin Gastroenterol Hepatol 2017;15:10611070.e1.

5. Ashton JJ, Borca F, Mossotto E, et al. Increased prevalence of anti-TNF therapy in paediatric inflammatory bowel disease is associated with a decline in surgical resections during childhood. Aliment Pharmacol Ther 2019;49:398-407.

6. Hossain A, Lördal M, Olsson AE, et al. Sustained clinical benefit, improved quality of life, and reduced intestinal surgery from maintenance infliximab treatment in inflammatory bowel disease. Scand J Gastroenterol 2020;55:178-83.

7. Lemaitre M, Kirchgesner J, Rudnichi A, et al. Association between use of thiopurines or tumor necrosis factor antagonists alone or in combination and risk of lymphoma in patients with inflammatory bowel disease. JAMA 2017;318:1679-86.

8. Borren NZ, van der Woude CJ, Ananthakrishnan AN. Fatigue in IBD: epidemiology, pathophysiology and management. Nat Rev Gastroenterol Hepatol 2019;16:247-59.

9. Barrett K, Saxena S, Pollok R. Using corticosteroids appropriately in inflammatory bowel disease: a guide for primary care. Br J Gen Pract 2018;68:497-8.

Competing interests: Neil Chanchlani is an associate editor for CMAJ and was not involved in the editorial decision-making process for this article. Richard Russell declares an NHS Research Scotland Senior Research Fellowship and speaker fees, travel support or participation in medical board meetings with Nestlé, AbbVie, Dr. Falk Pharma, Takeda, Napp, Mead Johnson, Nutricia and 4D Pharma. No other competing interests were declared.

Content licence: This is an Open Access article distributed in accordance with the terms of the Creative Commons Attribution (CC BY-NC-ND 4.0) licence, which permits use, distribution and reproduction in any medium, provided that the original publication is properly cited, the use is noncommercial (i.e., research or educational use), and no modifications or adaptations are made. See: https://creativecommons.org/ licenses/by-nc-nd/4.0/ 